\title{
Bioinformatics' Challenges to Computer Science: Bioinformatics Tools and Biomedical Modeling
}

\author{
Mario Cannataro ${ }^{1}$, Rodrigo Weber dos Santos ${ }^{2}$, and Joakim Sundnes ${ }^{3}$ \\ 1 University Magna Graecia, Catanzaro, Italy \\ cannataro@unicz.it \\ 2 Federal University of Juiz de Fora, Brazil \\ rodrigo.weber@ufjf .edu.br \\ 3 Simula Research Laboratory, Norway \\ sundnes@simula.no
}

\begin{abstract}
The second edition of the workshop on Bioinformatics' Challenges to Computer Science aimed to discuss the gap between bioinformatics tools and biomedical simulation and modeling. This short paper summarizes the papers accepted for the workshop, focusing on bioinformatics applications at the genomics and molecular level as well as simulation and management at the biomedical level, and gives a brief outlook on future developments.
\end{abstract}

Keywords: Bioinformatics, Data Management and Integration, Modelling and Simulation of Biological Systems, Data Visualization.

\section{Bioinformatics - An Overview}

Bioinformatics 12] is an interdisciplinary field linking biology with computer science. In the strictest interpretation of the term, see e.g. http://www.wikipedia.org, bioinformatics is defined as the application of computer science to advance the field of molecular biology. However, for this workshop we have adopted a broader definition, which covers development of advanced algorithms and computational tools to study biomedical problems in general. In addition to classical bioinformatics, this includes the development and use of mathematical models to describe the aggregate behavior of cells, tissues, organs and organ systems, a field often referred to as systems biology or integrative biology.

Bioinformatics is a field under rapid development, as improvements of both computer hardware, algorithms and data acquisition allow the construction and analysis of increasingly complex and detailed models and datasets. However, although substantial progress has been made, the potential benefit from computer science tools in biomedical science is huge, and still far from being fully utilized. Continuing along the track started with its first edition [3], the current edition of the workshop on Bioinformatics' Challenges to Computer Science aims to discuss the gap between bioinformatics tools and biomedical simulation and modeling.

G. Allen et al. (Eds.): ICCS 2009, Part I, LNCS 5544, pp. 807-809, 2009.

(C) Springer-Verlag Berlin Heidelberg 2009 


\section{Goals}

The aim of this workshop was to bring together scientists from computer and life sciences, to discuss future directions of research. As noted above, our broad definition of the bioinformatics term essentially divides current research into two categories; one dealing with data management and analysis on the genetic and molecular scale, and another dealing with development and application of advanced system level models. While both of these branches have obtained considerable progress in the past, and may continue to do so for many years to come, a closer interaction between the two groups hold an even larger potential.

\section{Workshop Summary}

The papers of the present workshop cover a broad range of applications. The first session is devoted to applications of simulation studies and signal analysis. Indolfi et al. focus on the role of real-time image processing in medicine and present a novel software tool able to assist the physician during pre-implant analysis and thus supporting an optimal stent choice. The paper by Peters et al. deals with the application of electrical impedance tomography to assess cardiac ejection fraction. Numerical methods based on biophysical models are used to analyze the potential of this tool. The third paper of the session, by Palumbo et al., describes the integrated analysis of different biomedical signals of the lower limb to characterize various physical exercises. Muscles activity and training effectiveness were evaluated by monitoring electromiography (EMG) signals, metabolic data, oxygen uptake and heart rate, with the overall aim to develop a system able to manage different information coming by various electronic devices.

The papers of the second session focus on the application of computer science tools in genetics and molecular biology. The paper by Zhang et al. focuses on an important research problem in DNA microarray data analysis, the discovery of gene co-regulatory relationships, and describes an improved algorithm for the analysis of gene co-regulation. Menif et al. address the Haplotype Assembly Problem (HAP), an important aspect of Single Nucleotide Polymorphisms (SNP) analysis, and present a set of algorithms for Minimum Fragment Removal based on the processing of strings. The paper by Miceli et al. addresses the protein structure prediction problem, central for the understanding of protein function, and compares several available automatic secondary structure prediction tools.

In addition to the listed papers, the workshop includes time slots for discussion of future directions in bioinformatics. These slots will be used for discussing potential future development of the presented research results, with particular focus on potential directions of mutual benefit and joint interest.

\section{Conclusions and Outlook}

Bioinformatics tools hold a huge potential for use in medical research and clinical practice. Both the analysis of genetic information offered by classical bioinformatics and the study of systems behavior with detailed mathematical models 
may lead to huge benefits for drug development and personalized health care. However, the full potential of the field can only be achieved by successfully combining these two branches of the field. Aquisition and analysis tools for genetic data may be combined with system level models to perform patient specific computer simulations of organs and organ systems. This link from the Genome to the Physiome (see for instance the NSR (National Simulation Resource) Physiome Project - http://nsr.bioeng.washington.edu/) has been defined as a future grand challenge of biomedical research, and is subject of substantial research world wide. In the discussion slots of the current workshop we will attempt to provide a link between the presented research results, and to identify potential collaborative projects that would help to bridge the gap between the two branches.

Acknowledgements. We would like to thank the members of the program committee for the invaluable assistance on reviewing the papers and the organizers of ICCS for promoting this workshop.

\section{References}

1. Jones, N.C., Pevzner, P.A. (eds.): An Introduction to Bioinformatics Algorithms. MIT Press, Cambridge (2004)

2. Cohen, J.: Bioinformatics - an introduction for computer scientists. ACM Computing Surveys 36, 122-158 (2004)

3. Cannataro, M., Romberg, M., Sundnes, J., Santos, R.W.d.: Bioinformatics' challenges to computer science. In: Bubak, M., van Albada, G.D., Dongarra, J., Sloot, P.M.A. (eds.) ICCS 2008, Part III. LNCS, vol. 5103, pp. 67-69. Springer, Heidelberg (2008) 